\title{
Optimized T1-MPRAGE Sequence for Better Visualization of Spinal Cord Multiple Sclerosis Lesions at 3T
}

\author{
G. Nair, M. Absinta, and D.S. Reich
}

\begin{abstract}
BACKGROUND AND PURPOSE: Spinal cord lesions are highly prevalent in MS, and their visualization can help both in diagnosis and patient follow-up. However, the sensitivity of MR imaging to spinal cord lesions remains poor, primarily because of suboptimal contrast between lesions and a normal-appearing cord. Here, we propose an optimized 3D MPRAGE sequence for improved detection of MS lesions in the spinal cord at 3T.
\end{abstract}

MATERIALS AND METHODS: Images were acquired by use of T2 FSE, STIR, T1-gradient recalled-echo (for T1 mapping), and T1-MPRAGE in the sagittal plane, and T2*-weighted scans in the axial plane, on 40 patients with MS and 7 healthy volunteers. Two observers qualitatively evaluated the images for lesion conspicuity. Lesions seen between the $\mathrm{Cl}$ and $\mathrm{C} 4$ segments in 10 randomly selected patients with MS were further evaluated quantitatively for contrast-to-noise ratio between the lesion and normal-appearing cord, and for lesion burden.

RESULTS: Spinal cord lesions were more conspicuous on the optimized T1-MPRAGE sequence than on any other sequence tested. Detailed analysis revealed that lesions were almost 3 times more conspicuous $(P<.01)$, and the total lesion volume was 2 times greater $(P<$ $.05, n=10$ ), in the T1-MPRAGE sequence compared with the standard STIR sequence. Correlation of clinical disability (Expanded Disability Status Score) with lesion load from each sequence also demonstrated the importance of the improved lesion conspicuity with T1-MPRAGE.

CONCLUSIONS: The optimized T1-MPRAGE sequence described here improves the reliability of lesion visualization and estimation of lesion burden, especially when used in conjunction with other well-established clinical sequences.

ABBREVIATIONS: CNR = contrast-to-noise ratio; EDSS = Expanded Disability Status Score; GRAPPA = generalized autocalibrating partially parallel acquisition; MERGE = multi-echo recombined gradient-echo; NAC = normal-appearing cord; PSIR = phase-sensitive inversion recovery; GRE = gradient recalled-echo

M $\mathrm{S}$ is a disease of the central nervous system, and spinal cord lesions are widely prevalent in patients with MS. It is thought that, in the proper context, the presence of cord lesions is highly specific for the diagnosis of $\mathrm{MS},{ }^{1-3}$ and several

Received December 28, 2012; accepted after revision February 18, 2013. From the National Institute of Neurological Disorders and Stroke (G.N., M.A., D.S.R.), National Institutes of Health, Bethesda, Maryland; Neuroimaging Research Unit (M.A.) and Department of Neurology, Institute of Experimental Neurology, Division of Neuroscience, San Raffaele Scientific Institute, Vita-Salute San Raffaele University, Milan, Italy; and Department of Radiology and Neurology (D.S.R.), Johns Hopkins University, Baltimore, Maryland.

Support: Intramural Research Program of NINDS.

Paper previously presented at: Annual Meeting of the Americas Committee for Treatment and Research in Multiple Sclerosis (ACTRIMS), May 30-June 2, 2012; San Diego, California.

Please address correspondence to Govind Nair, Translational Neuroradiology Unit, National Institute of Neurological Disorders and Stroke, National Institutes of Health; Bldg 10, Rm 5C103; 10 Center Dr, MSC 1400; Bethesda, MD 20892; e-mail: reichds@ninds.nih.gov

- Indicates open access to non-subscribers at www.ajnr.org

http://dx.doi.org/10.3174/ajnr.A3637 studies have shown spinal cord abnormalities in $75 \%-90 \%$ of patients clinically diagnosed with MS. ${ }^{4-7}$ Visualization of spinal cord lesions allows approximately $18 \%$ more patients to meet the McDonald dissemination in space criteria for the diagnosis of MS. ${ }^{8}$ Cord lesions are also believed to occur in isolation, further highlighting the importance of visualizing lesions in the spinal cord for the diagnosis of MS..$^{8-10}$

MR imaging of the spinal cord is challenging not only because of the small size of structures but also because of artifacts from respiration, cardiac, and CSF pulsation, and cord movement during the cardiac cycle. Although advances in MR imaging hardware and pulse sequences have mitigated some of these challenges, sensitivity to visualization of cord lesion remains poor. ${ }^{11,12}$ Several studies have proposed specialized and optimized sequences for improving lesion visualization in the spinal cord, especially the cervical cord, including double-echo spin-echo, ${ }^{13}$ STIR, ${ }^{14-16}$ phase-sensitive inversion recovery (PSIR), ${ }^{17}$ and multi-echo recombined gradient-echo (MERGE on GE scanners, also called Multi-Echo Data Image Combination or MEDIC on Siemens 
scanners and multi-echo Fast Field Gradient Echo or mFFE on Philips scanners). ${ }^{18-20}$ Of these, the proton density and T2 (double-echo) spin-echo or $\mathrm{FSE}^{21}$ and $\mathrm{STIR}^{14}$ sequences have been accepted as a clinical standard for spinal cord imaging in MS, ${ }^{13}$ primarily because of the ease of implementation on a wide range of scanners. However, lesions are demonstrably more conspicuous in sequences such as PSIR ${ }^{17}$ and MERGE. ${ }^{18}$ Furthermore, despite sequence improvements, the correlation between lesion volume and clinical Expanded Disability Status Score (EDSS) remains low. ${ }^{22,23}$ One contributing factor could be that MR imaging sequences are insensitive to many lesions in the cord, and that radiologic calculation of lesion burden is inaccurate. ${ }^{15}$

An optimized sequence for detection of cord lesions may improve diagnostic efficiency and accuracy of CNS lesion load estimation. The MPRAGE sequence offers a unique opportunity, through optimization of T1-contrast and SNR. Here, we qualitatively evaluate a T1-weighted MPRAGE sequence for efficiency of visualizing lesions in the cervical and thoracic spinal cord and quantitatively compare its performance with other sequences including STIR, T2-weighted, T2*-weighted, and noninversionprepared T1-weighted gradient-echo sequences in the upper cervical spinal cord.

\section{MATERIALS AND METHODS \\ Patients}

MR imaging of the cervical and thoracic spinal cord was performed on 40 patients and 7 healthy control participants. MR images from 10 patients with MS were randomly selected for a more detailed quantitative analysis of lesions in the upper cervical cord. Written, informed consent was obtained from all participants, and the Institutional Review Board at the National Institutes of Health approved all protocols.

\section{MR Imaging}

MR imaging was performed on a 3T Skyra system (Siemens, Erlangen, Germany) equipped with a 20-channel head-neck coil and a 16-channel spine-array coil. Standard clinical sequences and sequences optimized elsewhere that have been traditionally used to visualize spinal cord lesions such as T2-weighted FSE, STIR, T1-weighted gradient recalled-echo (GRE), and T2*-weighted GRE sequences were compared with the optimized T1-MPRAGE sequence in the cervical and thoracic regions separately.

In the cervical spine, T2-weighted images were acquired in the sagittal plane by use of a 2D FSE sequence (T2-FSE) with TR, 3500 $\mathrm{ms}$; TE, $102 \mathrm{~ms}$; 0.7-mm nominal in-plane resolution; and 1-mm section thickness (no intersection gap), with a scan time of 3 minutes 40 seconds. STIR images were acquired in the sagittal plane with a 2D inversion-prepared FSE sequence with TR, $4000 \mathrm{~ms}$; TE, $68 \mathrm{~ms}$; TI, $210 \mathrm{~ms}$; parallel imaging with generalized autocalibrating partially parallel acquisition (GRAPPA 2); 0.8-mm nominal in-plane resolution; 2-mm section thickness; a 10\% gap between sections; and a scan time of 4 minutes 30 seconds. A 3D-MPRAGE sequence (T1-MPRAGE) was acquired in the sagittal plane with TR, $3000 \mathrm{~ms}$; TE, $4.5 \mathrm{~ms}$; TI, $750 \mathrm{~ms}$; flip angle, $8^{\circ}$; parallel imaging (GRAPPA 2); 1-mm isotropic resolution; and a scan time of 7 minutes 30 seconds. T1 mapping was done by use of two 3D-gradient-echo sequences with TR, $7.8 \mathrm{~ms}$; TE, $3 \mathrm{~ms}$; 1-mm isotropic resolution; and flip angles of $3^{\circ}$ and $16^{\circ}$ (T1GRE), for a scan time of approximately 3 minutes per flip angle. Finally, $\mathrm{T} 2^{\star}$-weighted images were acquired in the axial plane with the 2D MEDIC sequence (T2*-GRE) with TR, $775 \mathrm{~ms}$; TE, 11 ms; flip angle, $20^{\circ}$; parallel imaging (GRAPPA 2); nominal inplane resolution, $0.58 \mathrm{~mm}$; section thickness, $5 \mathrm{~mm}$ (no intersection gap); and a scan time of 3 minutes 30 seconds. The protocols were very similar for imaging of the thoracic spine, except that the sequence was GRE, and field-of-view and acquisition matrix were modified to cover the entire thoracic spinal cord. The T1-GRE sequence was repeated postgadolinium (gadobutrol, $0.1 \mathrm{mmol} /$ $\mathrm{kg}$ ) only if clinically indicated.

\section{Data Analysis}

By consensus, a neurologist (M.A., with 7 years of experience) and a neuroradiologist (D.S.R., with 10 years of experience) rated each lesion from the MS and healthy control groups in a blinded fashion. Images obtained from all of the sequences were assessed simultaneously, and lesions that were conspicuous in any of the pulse sequences were counted, and their vertebral levels noted for each patient. For detailed quantitative analysis, scans from 10 patients were randomly selected from the MS group. 3D scans were reformatted only in orthogonal (axial, coronal, and sagittal) planes in OsiriX (http://www.osirix-viewer.com/) ${ }^{24}$ for qualitative evaluation, and a lesion was labeled confirmed if it was present in more than 1 sequence or if it had a characteristic appearance on reformation. None of the quantitative analysis used curved or oblique reformatting. The conspicuity of each lesion in the various pulse sequences was scored as being absent or of poor, good, or excellent conspicuity (scores from 0-3). Lesion detection efficiency for each sequence was calculated as an average percent of lesions detected with good or excellent conspicuity to the total number of lesions seen in each patient. T1 maps were calculated on a pixel-by-pixel basis in Matlab (MathWorks, Natick, Massachusetts) by use of the variable flip-angle GRE scans, described elsewhere. ${ }^{25}$ ROIs were drawn on visible lesions (L) and normal-appearing brain stem or cord (NAC) at the C1 level in each sequence, and the contrast-to-noise ratios (CNRs) were calculated with the formula $\left(S_{L}-S_{N A C}\right) /\left(S_{L}+S_{N A C}\right)$, where $S$ is a signal in an ROI. Lesion volumes in the $\mathrm{C} 1-\mathrm{C} 4$ region were determined from each patient on the basis of conspicuity (only lesions of good or excellent conspicuity) in each imaging sequence, and the total lesion volume was normalized and compared with that derived from a STIR sequence (paired $t$ test). The lesion volumes from each sequence were also correlated with EDSS.

\section{RESULTS}

Of the 40 patients studied, 1 patient was diagnosed with neuromyelitis optica, and the data from this patient were not analyzed. Detailed demographic information is shown in the accompanying Table. In the blinded analysis, a total of 324 lesions were identified in the spinal cords of 39 patients with MS; no lesions were identified in the healthy volunteer group. Typical lesions in the cervical cord from 2 different participants (top and bottom row) are shown in Fig 1. Focal and diffuse lesions in the cervical spinal cord could be clearly and consistently visualized in the T1-MPRAGE 
Demographic data for participant groups

\begin{tabular}{|c|c|c|c|c|c|}
\hline Group & No. of Patients (Sex) & Age (y) & Diagnosis (MS Subtype) & Disease Duration (y) & EDSS (Range) \\
\hline Healthy volunteers & $7(5 F)$ & $44 \pm 7$ & - & - & - \\
\hline All patients with MS & $39(21 \mathrm{~F})^{a}$ & $49 \pm 12$ & $\begin{array}{l}\text { Relapsing-remitting }(n=14) \\
\text { Secondary-progressive }(n=10) \\
\text { Primary-progressive }(n=15)\end{array}$ & $13 \pm 11$ & $1.0-7.0$ \\
\hline MS subgroup for quantitative analysis & $10(9 \mathrm{~F})$ & $47 \pm 12$ & $\begin{array}{l}\text { Relapsing-remitting }(n=4) \\
\text { Secondary-progressive }(n=5) \\
\text { Primary-progressive }(n=1)\end{array}$ & $17 \pm 14$ & $1.0-6.5$ \\
\hline
\end{tabular}

${ }^{a}$ One of 40 patients recruited was dropped from the analysis when clinically diagnosed with neuromyelitis optica.
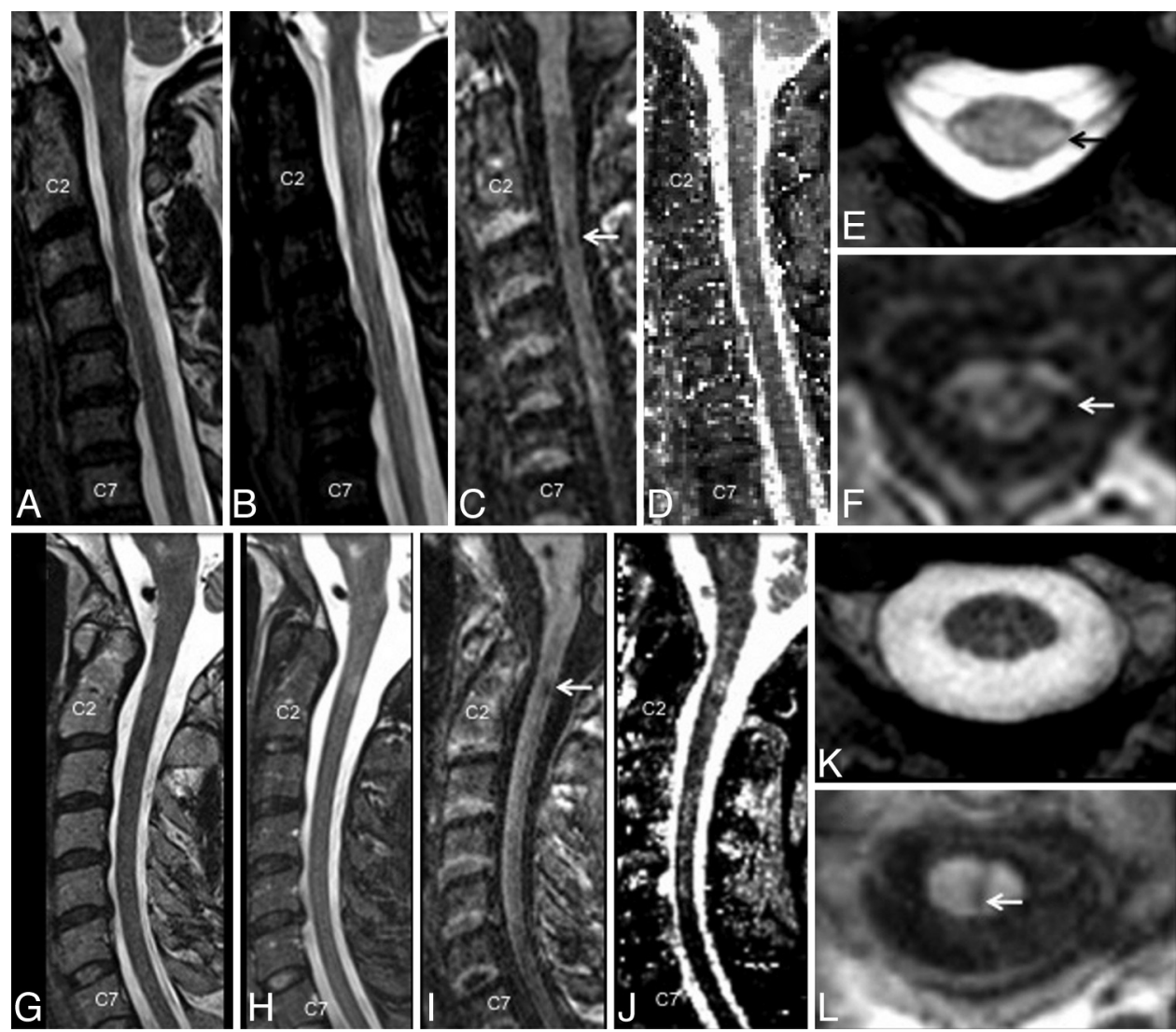

FIG 1. A comparison of imaging techniques for visualization of cervical cord lesions in a 45-year-old woman ( $A-F)$ and a 43 -year-old man ( $G-L)$, both with relapsing-remitting MS. T1-weighted MPRAGE ( $C$ and $I$ ) performed better than routine clinical T2-weighted FSE ( $A$ and $G)$, STIR $(B$ and $H)$, and quantitative T1 maps of the cervical cord ( $D$ and $J)$, generated by use of a multiple flip angle gradient-echo sequence. The lesions were also visible on axially reformatted T1-MPRAGE images $(F$ and $L)$ and in these cases were also well visualized on axial T2*-GRE $(E$ and $K)$.

sequence (Fig $1 C,-F,-I,-L)$. T2*-GRE images were consistently good at visualizing lesions with some portion extending into the cord (Fig $1 E$ ) but performed more poorly for lesions on the surface of the cord (Fig $1 K$ ). Lesions were consistently less conspicuous on the STIR and T2-FSE images (Fig $1 A,-B,-G$ ).

Similar results were observed from the thoracic spine, with most lesions being conspicuous on the T1-MPRAGE images followed by T2*-GRE, STIR, and T2-FSE (Fig 2). T1-MPRAGE was able to detect acute lesions as well (Fig $3 A-C)$. Neither the T1MPRAGE nor the other scans from the healthy control participants showed any focal hypointense lesions (Fig 3D) such as those seen in the patients with MS. Coronal and curved reformatted (used only for visualization in this figure and not for quantitative analysis) T1-MPRAGE of the cervical cord in patients with MS (Fig 4A, -B) depicts the lesions mainly on the surface of the cord. A plot of average incidence of lesions that were conspicuous in any of the pulse sequences, as a function of vertebral body level, is shown in Fig 4C. Forty percent of all lesions occurred in the C1-C4 segments; therefore, these levels were chosen for detailed quantitative analysis.

Quantitative analysis from 10 patients with MS showed that the CNR between the lesion and NAC was $0.5 \pm 0.12$ for T1MPRAGE, $0.15 \pm 0.07$ for STIR, $0.13 \pm 0.07$ for T2-FSE, and $0.08 \pm 0.03$ for T2*-GRE (Fig $5 A,{ }^{* *} P<.01$, unpaired $t$ test). The T1 maps calculated from the 2-flip angle method yielded a T1 of $1749 \pm 260 \mathrm{~ms}($ mean $\pm \mathrm{SD})$ in the lesions compared with $1027 \pm$ $193 \mathrm{~ms}$ in the NAC, yielding a CNR of $0.25 \pm 0.08$, which was significantly lower than that of the T1-MPRAGE sequence.

In the C1-C4 segments, the T1-MPRAGE sequence detected 59 of the 60 observed lesions that were judged to have good or excellent conspicuity by the 2 expert observers, resulting in a lesion detection efficiency of $98 \%$ per patient (Fig $5 B$; $n=10$ pa- 

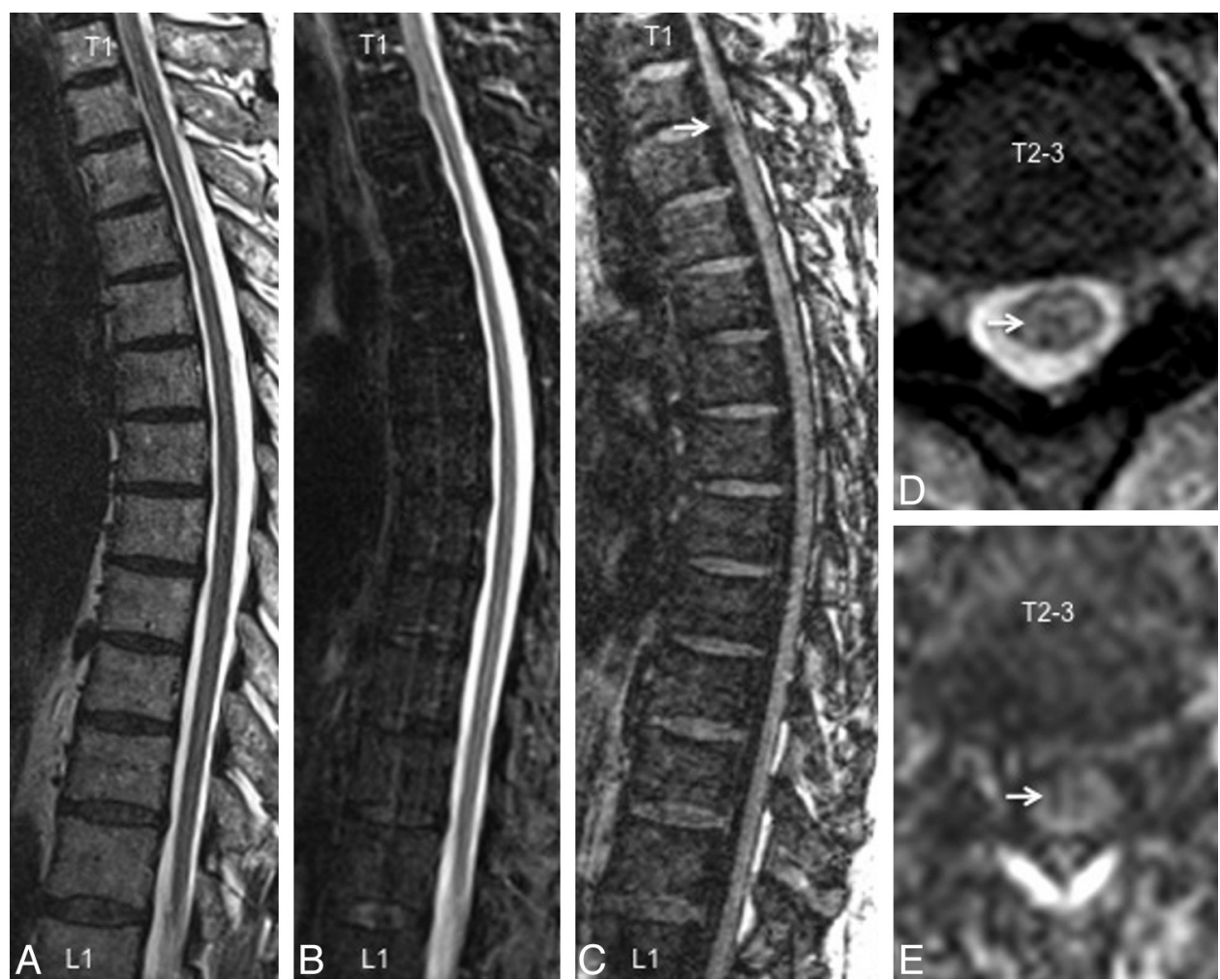

FIG 2. A comparison of imaging techniques for visualization of thoracic cord lesions in a 45-year-old woman with relapsing-remitting MS. Just as in the cervical cord, T1-weighted MPRAGE (C) performed better than routine clinical T2-weighted FSE $(A)$, STIR (B), and T2*-GRE (D) for visualization of lesions. The lesion denoted by an arrow has a typical appearance and could be confirmed on axially reformatted T1-MPRAGE images (E).
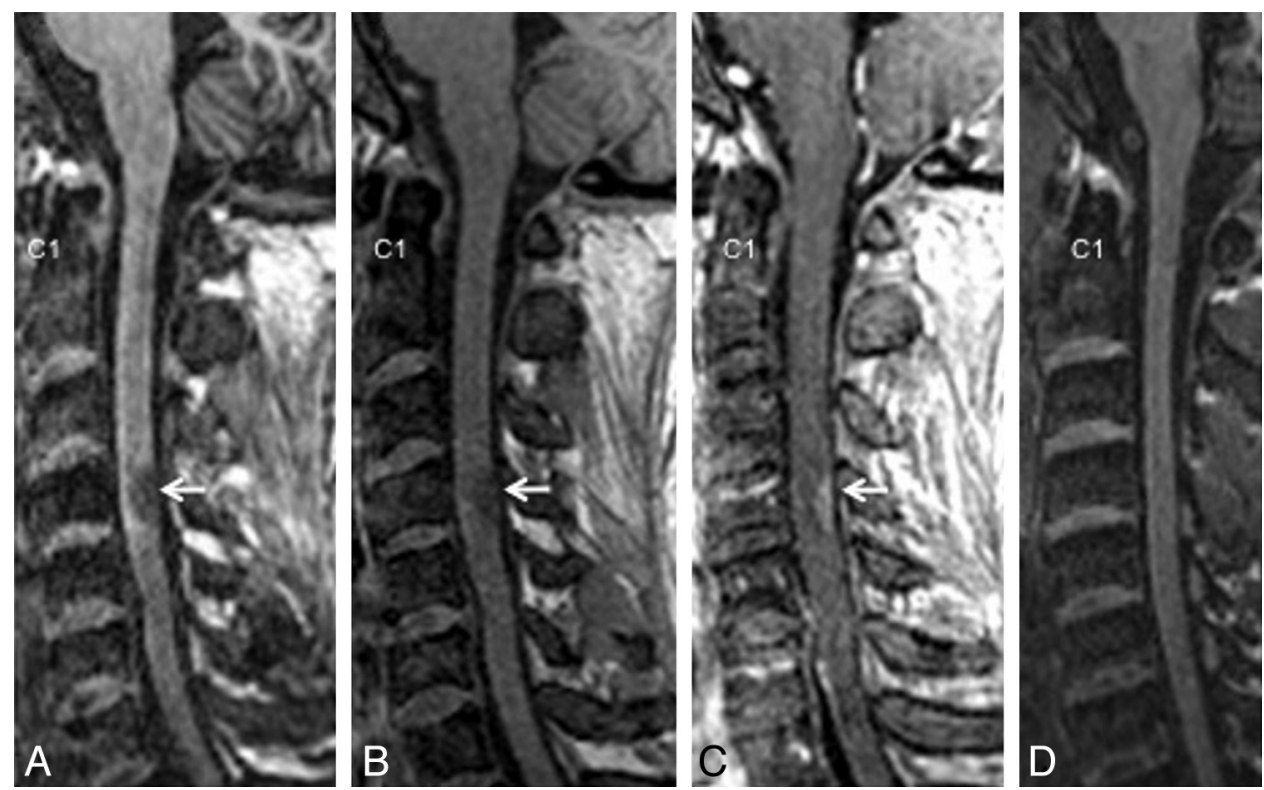

FIG 3. An acute spinal cord lesion in the $C 4$ region (arrow, 31-year-old man) as seen in T1-MPRAGE (A), precontrast (B), and postcontrast (C) T1-weighted gradient-echo $(C)$ images. (D) T1-MPRAGE from a healthy control participant (38-year-old man) for comparison.

tients). Forty-five of the 60 lesions were conspicuous in the axial $\mathrm{T}^{*}$ sequences $(75 \%$ of the lesions; lesions detected in $82 \%$ of the patients with cord lesions). The average length of lesions judged to have excellent conspicuity was $7.2 \pm 0.6 \mathrm{~mm}$ in the inferior-superior direction, compared with $5.2 \pm 0.6 \mathrm{~mm}$ for lesions judged to be less conspicuous ( $P=.02$; lengths calculated on the T1MPRAGE sequence). Only 26 of the 60 lesions were conspicuous on the STIR ( $43 \%$ of the lesions; lesions detected in $51 \%$ of the patients with cord lesions), and only 15 were conspicuous on the T2-FSE sequences (25\% of the lesions; lesions detected in 32\% of the patients with cord lesions).

Average lesion volume from the T1-MPRAGE sequence was more than twice that calculated from the clinical standard STIR sequence and was significantly higher than that determined from 

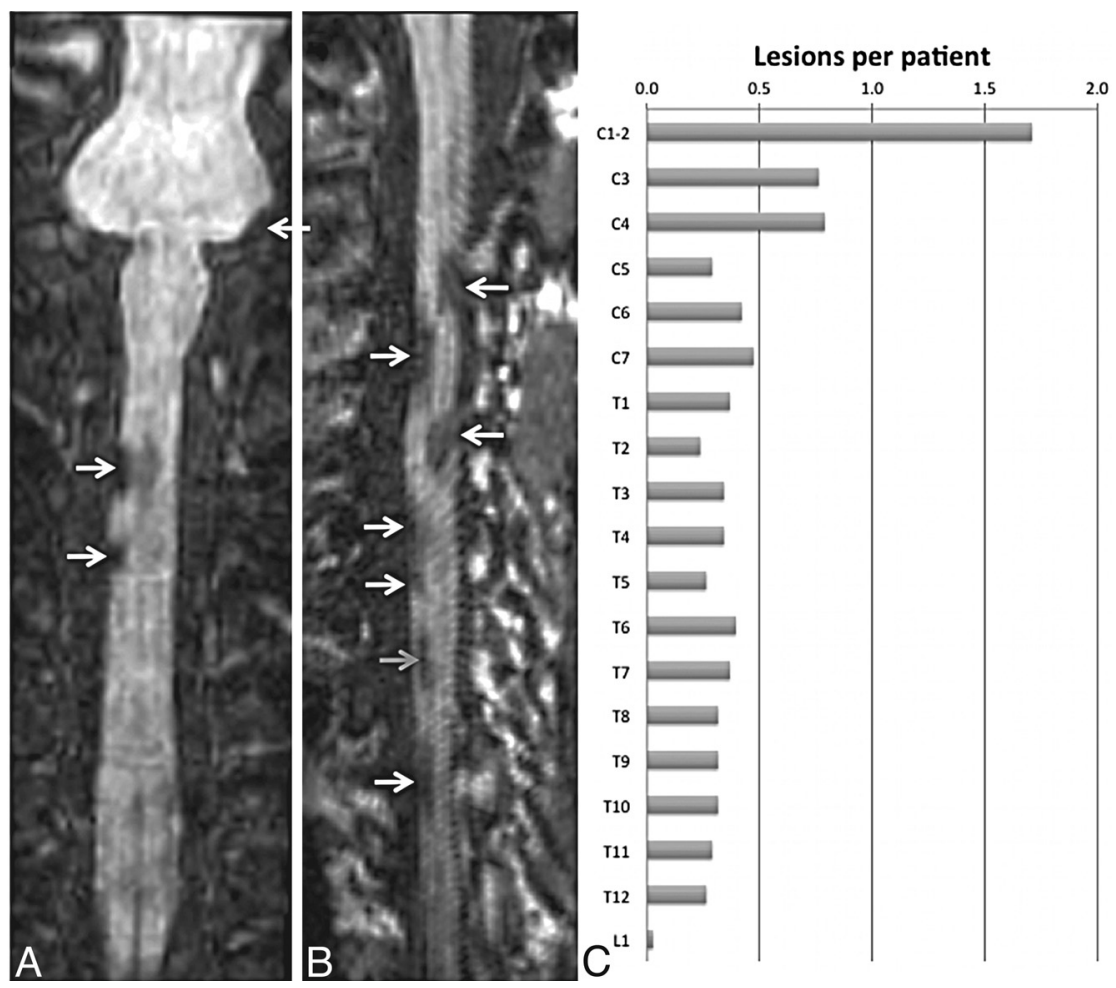

FIG 4. Coronal $(A)$ and curved-reformatted $(B)$ images from the Tl-weighted MPRAGE sequence in the cervical cord of 2 patients, showing lesions mainly on the surface of the cord (white arrows) and a lesion that appears to be completely within the cord (gray arrow). C, Incidence of lesions per vertebral body segment derived from all scans acquired in the MS population studied herein (Table, 39 patients with MS).

all other sequences $(P<.05 ; n=10$ patients). Lesion volume, calculated relative to the STIR sequence, was 1.95 for T1-MPRAGE, 1.67 for T2*-GRE, and 0.6 for T2-FSE $(P<.05$ by paired $t$ test; Fig $5 C)$. The smallest conspicuous lesion was $11.8 \mathrm{~mm}^{3}$ in the $\mathrm{T} 1-$ MPRAGE sequence, $16.6 \mathrm{~mm}^{3}$ in the T2*-GRE, $21.3 \mathrm{~mm}^{3}$ in the T2-FSE, and $24.8 \mathrm{~mm}^{3}$ in the STIR. Pearson correlation coefficient, determined between EDSS and lesion volume, approached significant levels only in the T1-MPRAGE (Pearson $r=0.52$; 1 -tailed $P=.06$; Fig $5 D$ ), and the correlation coefficients were larger in sequences that had better lesion conspicuity (T1MPRAGE and T2*-GRE).

\section{DISCUSSION}

The optimized T1-MPRAGE sequence was better at lesion visualization in both the cervical and thoracic spinal cord than the STIR, T2-FSE, T1-GRE, and T2*-GRE sequences tested herein. The optimized T1-MPRAGE sequence was able to reliably and distinctly detect both acute and chronic lesions in the cord. The high CNR of the optimized T1-MPRAGE played an important role in lesion detection. Reformatting the images in the axial and coronal planes proved to be useful in identification of the lesions, which was made easier because of the high isotropic resolution of the T1-MPRAGE.

It should also be noted that the central canal of the spinal cord was hypointense compared with the NAC in the T1-MPRAGE, and there was subtle hypointensity in the areas surrounding the central canal. Such signal changes are commonly seen in other high-resolution sequences, as well as in the healthy control participants (Fig 3D). However, the signal in the confirmed lesions was distinctly more hypointense and focal than these other signal changes and could easily be characterized as such (Figs 1 and 2). Only 1 of 60 confirmed lesions was of poor conspicuity on the T1-MPRAGE sequence.

\section{Comparison of Sequences}

Most lesions (45/60, or $75 \%$ ) were also conspicuous on the $\mathrm{T} 2{ }^{\star}$-GRE images. The lesions that were conspicuous on the $\mathrm{T} 2^{\star}$-GRE had a significantly larger extent in the superior-inferior direction than those that were inconspicuous on the T2*-GRE, emphasizing the role of partial volume averaging and image resolution in the detection of lesions. However, it must be noted that 7 of the 15 lesions that were inconspicuous on the T2*-GRE were larger than $5 \mathrm{~mm}$ in the superior-inferior direction, indicating that other factors, such as proximity to CSF (eg, Fig $1 K$ ) and motion or pulsation artifacts, can be detrimental to lesion visualization on $\mathrm{T} 2^{\star}$-GRE. Several improvements have been suggested to the T2*-GRE sequence, including higher resolution, $3 \mathrm{D}$ acquisition, and magnetization transfer preparation, all of which could improve the conspicuity of MS lesions; these were not explored herein. ${ }^{20}$ Indeed, not every lesion was better seen on the T1MPRAGE, and a few were even better depicted in the T2*-GREsequences, as can be seen in Fig 1 (top row). Nevertheless, more lesions with typical MS configuration were appreciated on the T1-MPRAGE overall. The T1-MPRAGE sequence may therefore be used in conjunction with other well-established clinical sequences, such as T2*-GRE, to improve diagnostic confidence.

The T2-FSE sequence was optimized for T2 contrast and resolution but proved to be inadequate for visualization of lesions. As many as $45(75 \%)$ of the 60 lesions were invisible or were of poor conspicuity on the T2-FSE sequence. It is conceivable that the TE used herein is suboptimal for lesion detection in the spinal cord, and that lesions may be more conspicuous in proton attenuation-weighted conventional spin-echo images such as those described elsewhere for diffuse lesions. ${ }^{14,16}$ However, such sequences were not explored herein because of potential motion artifacts, low resolution, and total scan time considerations. The STIR sequence has long been known to be useful for lesion detection, ${ }^{15,16,26,27}$ and a standard clinical fast-STIR sequence with an inversion time of $200 \mathrm{~ms}$ was used here. The STIR sequence did indeed perform better than T2-FSE in both CNR and lesion visualization, as expected, despite the larger section thickness and a gap between sections.

Others have calculated the lesion-to-cord CNR from various sequences as a normalized difference in signal intensity from the lesion to cord. Hittmair et al ${ }^{14}$ demonstrated a $187 \%$ increase in lesion-to-cord CNR with the STIR sequence compared with routine T2-FSE, by using a 1.5T scanner with lower 

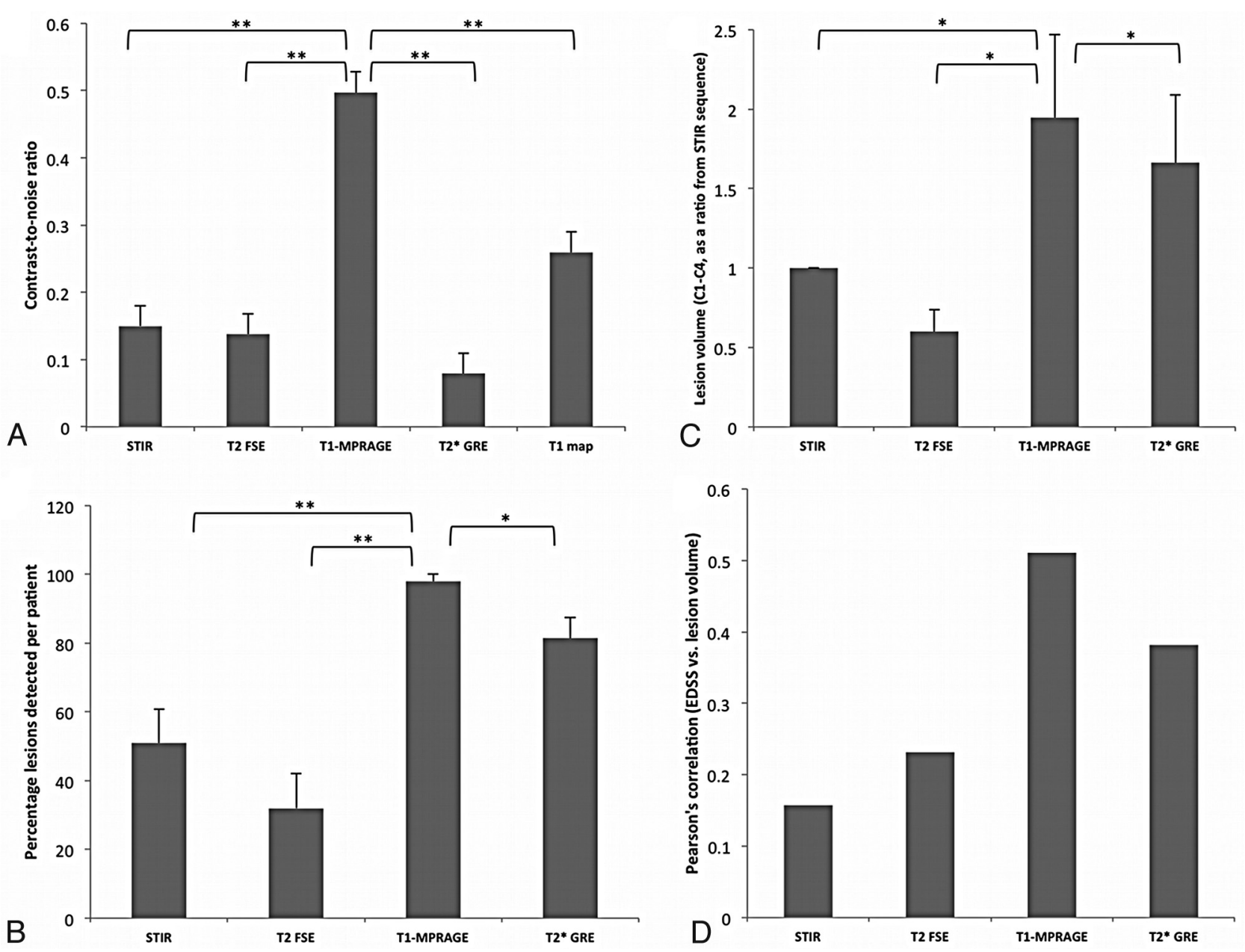

FIG 5. CNR $(A)$, percentage of lesions detected per patient ( $B$ ), lesion volume (in $\mathrm{mm}^{3}$ ) between the $\mathrm{Cl}$ and $\mathrm{C} 4$ vertebral body segments $(C)$, and Pearson correlation coefficient between lesion load and EDSS from 10 participants obtained by use of various pulse sequences for imaging cord lesions. The CNR and lesion volume calculated from the T1-MPRAGE sequence were significantly higher than other sequences tested $\left({ }^{\star} P<.05\right.$, $\star * P<.01$, paired $t$ test).

spatial resolution. The CNR from the $\mathrm{T} 2^{\star}$-MERGE sequence was reported to be approximately $200 \%$ higher than the T2FSE sequence in the study by White et al, ${ }^{18}$ and Poonawalla et $\mathrm{al}^{17}$ demonstrated an approximately $200 \%$ increase in the CNR in STIR compared with T2-FSE at 3T. This result is similar to the observation herein of increased lesion conspicuity in the T2*-GRE and STIR images compared with the T2-FSE images. The formula for the CNR used herein normalized its value between 0 and 1 , with 1 being the best contrast. Repeating the calculation by using formulas similar to the prior reports, on the data presented herein, shows a $190 \%$ increase in the CNR in STIR compared with T2-FSE, and a further $180 \%$ increase in CNR in T1-MPRAGE compared with STIR. However, the difference in CNR between T2*-GRE and T2-FSE was far lower than reported elsewhere, ${ }^{18}$ probably because of differences in the pulse sequence parameters such as image resolution and the axial vs sagittal section plane. Furthermore, the T2*-GRE sequence herein used a section thickness of $5 \mathrm{~mm}$ for adequate whole-spine coverage in a reasonably short scan time, as opposed to $3 \mathrm{~mm}$ in prior reports. This larger section thickness, which engenders more partial volume averaging, may contribute to the reduced CNR seen here; reducing the section thick- ness could potentially increase the lesion detection rate at the cost of reduced SNR and increased scan time. As noted previously, the thick sections used in the T2*-GRE sequence made it difficult to pick up small lesions, especially on the surface of the cord (because of proximity to bright CSF). Despite these shortcomings, the availability of both the axially acquired T2*-GRE scans, with high in-plane resolution, as well as the isotropic T1-MPRAGE scans that can be reformatted in any plane, acts to substantially improve confidence in lesion detection and discrimination.

In a technique known as PSIR, reconstruction of real images (rather than magnitude images) in an inversion-prepared 2D gradient-echo sequence has been shown to be useful in the detection of cord lesions. ${ }^{17}$ We did not observe any immediate advantage of reconstructing the real component with the optimized T1MPRAGE sequence, mainly because the signal from the lesion was close to null. This modification is very easily implemented on most MR imaging scanners if needed.

\section{Technical Considerations}

The T1-MPRAGE pulse sequence has been well described and is now commonly used in clinical imaging of the brain. ${ }^{28}$ As 
demonstrated here, this sequence offers a unique opportunity to visualize lesions in the cord, as it offers ways to optimize the imaging contrast by using inversion pulses. However, the $3 \mathrm{D}$ acquisition technique is more susceptible to motion artifacts. Use of a spine array coil and parallel imaging enabled improved SNR while reducing artifacts, particularly in the cervical region. Sagittal acquisition, rather than coronal or axial sections, allowed coverage of the spinal cord in the least amount of time without having to change the acquisition matrix in a subjectsize-dependent manner. SNR of the T1-MPRAGE was boosted by increasing the TR as well as the flip angle, while at the same time maintaining the contrast between lesions and the normal-appearing spinal cord.

$3 \mathrm{D}$ acquisition allowed for high isotropic resolution and more accurate calculation of lesion load. Isotropic high-resolution images can also be reformatted in different planes for better visualization of lesions, especially those on the surface of the cord. ${ }^{6}$ However, 3D acquisition also makes the images more susceptible to movement artifacts, which is especially relevant for the spinal cord. Spinal cord MR imaging is plagued with artifacts from respiration, swallowing, and cardiac movement, as well as from movement of the cord during a cardiac cycle. Placing a saturation pulse anterior to the vertebral bodies can be considered to eliminate most of the movement artifacts from outside the cord. Movement of the cord itself was not found to be a major source of artifacts, however. Finally, a large acquisition matrix and small bandwidth helped mitigate truncation artifacts in the spinal cord.

\section{Study Limitations}

The major limitation of this study was the lack of a reference standard for lesion presence, because such data would require postmortem pathologic examination. In the absence of a pathologic reference standard, we used consensus between experienced observers as a radiologic reference standard, and we compared our results from T1-MPRAGE with STIR, which is the accepted standard for sagittal imaging. An additional limitation was that we only performed detailed, quantitative, volumetric analysis in a fraction of the cases; however, the qualitative analysis in the larger cohort suggests that the results from those cases would have generalized. A further limitation was that the quantitative analysis focused only on the upper cervical cord, rather than the whole cord; this region was chosen because prior studies ${ }^{6,7}$ have demonstrated that roughly $60 \%$ of spinal cord lesions occur in the cervical cord. Indeed, in the patient population studied herein, the $\mathrm{C} 1-\mathrm{C} 4$ segments accounted for $40 \%$ of all lesions seen in the spinal cord. Moreover, lesions in the WM of the upper cervical cord can affect both the upper and lower limbs, potentially improving correlations with disability scores, which were moderate in the analysis reported here. Further analysis could entail correlation of lesion burden along specific tracts from the entire cord with individual functional systems scores, including sensory, motor, and bowel/bladder. ${ }^{5}$ Such studies would require a larger patient cohort. Finally, the protocol parameters used for the T1MPRAGE could not differentiate between the GM and WM in the cord, thereby necessitating the use of additional sequences for complete characterization of lesion location.

\section{CONCLUSIONS}

The optimized T1-MPRAGE sequence was more efficient in the visualization of spinal cord lesions compared with other standard clinical sequences. EDSS correlated better with cord lesion volumes calculated from the T1-MPRAGE sequence than from the other sequences, suggesting that a substantial proportion of the lesion burden in the spinal cord has been missed in prior studies. Therefore, T1-MPRAGE can help in a more accurate estimation of the lesion load and, when used in conjunction with other sequences such as T2*-GRE and STIR, can increase diagnostic confidence through better visualization of these lesions.

\section{ACKNOWLEDGMENTS}

This study was supported by the Intramural Research Program of the National Institute of Neurologic Disorders and Stroke (NINDS), National Institutes of Health. We also thank the National Institute of Mental Health (NIMH)/NINDS Functional Magnetic Resonance Facility, John Ostuni (NINDS), Souheil Inati (NIMH), and the Neuroimmunology Branch clinical group for their help.

\section{REFERENCES}

1. Bot JC, Barkhof F, Lycklama a Nijeholt G, et al. Differentiation of multiple sclerosis from other inflammatory disorders and cerebrovascular disease: value of spinal MR imaging. Radiology 2002;223:46-56

2. Lycklama à Nijeholt GJ, Barkhof F, Scheltens P, et al. MR of the spinal cord in multiple sclerosis: relation to clinical subtype and disability. AJNR Am J Neuroradiol 1997;18:1041-48

3. Simon JH. The contribution of spinal cord MRI to the diagnosis and differential diagnosis of multiple sclerosis. J Neurol Sci 2000;172 Suppl 1:S32-35

4. Ikuta F, Zimmerman HM. Distribution of plaques in seventy autopsy cases of multiple sclerosis in the United States. Neurology 1976;26:26-28

5. Nijeholt GJ, van Walderveen MA, Castelijns JA, et al. Brain and spinal cord abnormalities in multiple sclerosis. Correlation between MRI parameters, clinical subtypes and symptoms. Brain 1998; 121(Pt 4):687-97

6. Weier K, Mazraeh J, Naegelin Y, et al. Biplanar MRI for the assessment of the spinal cord in multiple sclerosis. Mult Scler 2012; 18:1560-69

7. Qiu W, Raven S, James I, et al. Spinal cord involvement in multiple sclerosis: a correlative MRI and high-resolution HLA-DRB1 genotyping study. J Neurol Sci 2011;300:114-19

8. Bot JC, Barkhof F, Polman CH, et al. Spinal cord abnormalities in recently diagnosed MS patients: added value of spinal MRI examination. Neurology 2004;62:226-33

9. Thorpe JW, Kidd D, Moseley IF, et al. Spinal MRI in patients with suspected multiple sclerosis and negative brain MRI. Brain 1996; 119(Pt 3):709-14

10. Brex PA, O'Riordan JI, Miszkiel KA, et al. Multisequence MRI in clinically isolated syndromes and the early development of MS. Neurology 1999;53:1184-90

11. Vaithianathar L, Tench CR, Morgan PS, et al. Magnetic resonance imaging of the cervical spinal cord in multiple sclerosis-a quantitative T1 relaxation time mapping approach. J Neurol 2003; 250:307-15

12. Barkhof $F$. The clinico-radiological paradox in multiple sclerosis revisited. Curr Opin Neurol 2002;15:239-45

13. Simon JH, Li D, Traboulsee A, et al. Standardized MR imaging protocol for multiple sclerosis: Consortium of MS Centers consensus guidelines. AJNR Am J Neuroradiol 2006;27:455-61 
14. Hittmair K, Mallek R, Prayer D, et al. Spinal cord lesions in patients with multiple sclerosis: comparison of MR pulse sequences. AJNR Am J Neuroradiol 1996;17:1555-65

15. Philpott $C$, Brotchie P. Comparison of MRI sequences for evaluation of multiple sclerosis of the cervical spinal cord at 3 T. Eur J Radiol 2011;80:780-85

16. Thorpe JW, MacManus DG, Kendall BE, et al. Short tau inversion recovery fast spin-echo (fast STIR) imaging of the spinal cord in multiple sclerosis. Magn Reson Imaging 1994;12:983-89

17. Poonawalla AH, Hou P, Nelson FA, et al. Cervical spinal cord lesions in multiple sclerosis: T1-weighted inversion-recovery MR imaging with phase-sensitive reconstruction. Radiology 2008;246:258-64

18. White ML, Zhang Y, Healey K. Cervical spinal cord multiple sclerosis: evaluation with $2 \mathrm{D}$ multi-echo recombined gradient echo MR imaging. J Spinal Cord Med 2011;34:93-98

19. Martin N, Malfair D, Zhao Y, et al. Comparison of MERGE and axial T2-weighted fast spin-echo sequences for detection of multiple sclerosis lesions in the cervical spinal cord. AJR Am J Roentgenol 2012;199:157-62

20. Ozturk A, Aygun N, Smith SA, et al. Comparison of axial MR sequences for the detection of multiple sclerosis lesions in the cervical spinal cord at 3 T. In: Proceedings of the Annual Meeting of the American Society of Neuroradiology, New Orleans, Louisiana, June 2-5, 2008:316

21. Bot JC, Barkhof F, Lycklama a Nijeholt GJ, et al. Comparison of a conventional cardiac-triggered dual spin-echo and a fast STIR sequence in detection of spinal cord lesions in multiple sclerosis. Eur Radiol 2000;10:753-58

22. Stankiewicz JM, Neema M, Alsop DC, et al. Spinal cord lesions and clinical status in multiple sclerosis: A $1.5 \mathrm{~T}$ and $3 \mathrm{~T}$ MRI study. J Neurol Sci 2009;279:99-105

23. Filippi M, Paty DW, Kappos L, et al. Correlations between changes in disability and T2-weighted brain MRI activity in multiple sclerosis: a follow-up study. Neurology 1995;45:255-60

24. Rosset A, Spadola L, Ratib O. OsiriX: an open-source software for navigating in multidimensional DICOM images. J Digit Imaging 2004;17:205-16

25. Deoni SC, Rutt BK, Peters TM. Rapid combined T1 and T2 mapping using gradient recalled acquisition in the steady state. Magn Reson Med 2003;49:515-26

26. Mascalchi M, Dal Pozzo G, Bartolozzi C. Effectiveness of the short TI inversion recovery (STIR) sequence in MR imaging of intramedullary spinal lesions. Magn Reson Imaging 1993;11:17-25

27. Campi A, Pontesilli S, Gerevini S, et al. Comparison of MRI pulse sequences for investigation of lesions of the cervical spinal cord. Neuroradiology 2000;42:669-75

28. Deichmann R, Good CD, Josephs O, et al. Optimization of 3-D MPRAGE sequences for structural brain imaging. Neuroimage 2000;12: 112-27 